\title{
Existence and regularity of weak solutions to a model for coarsening in molecular beam epitaxy
}

\author{
JUN ZHANG* \\ Department of Mathematics, \\ Zhejiang University of Technology \\ Hangzhou 310032, Zhejiang \\ P. R. China, \\ and \\ PEICHENG ZHU ${ }^{1,2 \dagger}$ \\ ${ }^{1}$ Basque Center for Applied Mathematics (BCAM) \\ Building 500, Bizkaia Technology Park \\ E-48160 Derio, Spain \\ 2 IKERBASQUE, Basque Foundation for Science \\ E-48011 Bilbao, Spain
}

\begin{abstract}
Taking into account the occurrence of a zero of the surface diffusion current and the requirement of the Ehrlich-Schwoebel effect, Siegert et al [23] formulate a model of Langevin type that describes the growth of pyramidlike structures on a surface under conditions of molecular beam epitaxy, and that the slope of these pyramids is selected by the crystalline symmetries of the growing film. In this article, the existence and uniqueness of weak solution to an initial boundary value problem for this model is proved, in the case that the noise is neglected. The regularity of the weak solution to models, with/without slope selection, is also investigated.
\end{abstract}

Keywords. Ehrlich-Schwoebel effect, Model for coarsening dynamics, Molecular beam epitaxy, Slope selection, Existence weak solutions

MSC 2000. 35K55, 74E15.

Runing title: Solutions to a model for coarsening dynamics.

\section{Introduction}

Many processes occur mainly at surfaces of materials, such processes include crystal growth, catalytic reactions, production of nano-structures. Thus surfaces are of great technological and fundamental interest. There has been increasing interest in the understanding of the kinetics of surface growth processes, see e.g. [14, 2]. The growth of a

\footnotetext{
${ }^{*}$ E-mail: mathzj@zjut.edu.cn

${ }^{\dagger}$ E-mail: zhu@bcamath.org
} 
crystalline film from a molecular or atomic beam is commonly referred to as molecular beam epitaxy (MBE), which is among the most fined methods for the growth of thin solid films and is of great importance for applied studies [9]. MBE takes place in high vacuum or ultra high vacuum, for instance, some $10^{-8} \mathrm{~Pa}$. The most important aspect of MBE is the slow deposition rate that is typically less than $1000 \mathrm{~nm}$ per hour, so MBE allows the films to grow epitaxially. The slow deposition rates require proportionally better vacuum to achieve the same impurity levels as other deposition techniques. In turn, it is possible by using this technique to grow high-quality crystalline materials and form structures with very high precision in the vertical direction.

The mathematical theory has been developed since Langevin who proposed the equation named after him. The model by Kardar, Parisi abd Zhang (KPZ) [10] describes well growth process such as the Eden process [5], ballistic deposition [26], and growth of various restricted solid-on-solid models [19]. Thus the KPZ model has been widely accepted as a model for the growth of crystals, and also has been extended to various cases. Let us mention especially the following work: In [9] the authors consider the system that has potential barriers near step edges that suppress the diffusion of adatoms to a lower terrace. This effect is now commonly called Ehrlich-Schwoebel effect. They take into account the step-flow regime and instability, and proposed a continuum equation to model the growth in MBE, which is valid only at the early time as long as the slopes are much smaller than 1 . However this is too restrictive to describe the unstable three-dimensional growth of real materials in the later time regime. In order to take into account the occurrence of a zero in the surface diffusion current and the requirement of Ehrlich-Schwoebel effect, Seigert et al [23] thus introduce a current for a structure with a cubic symmetry so that the current is changed to the one that has a zero differing from 0 so that the model can be also applied to the regime when the slope is much greater than 1. In this article, we shall study this model, also the model without slope selection will also be investigated. Due to the forming of steps, pyramidlike surfaces, etc. during the crystal growth, it is more natural to assume that the initial data is in $L^{2}(\Omega)$ or $H^{1}(\Omega)$ than in $H^{2}(\Omega)$.

To formulate the model, we need some notations. Let $x=\left(x_{1}, x_{2}\right) \in \Omega \subset \mathbb{R}^{2}$ be material point, $\Omega$ is an open bounded set with smooth boundary $\partial \Omega$. Let $t$ be the time variable. $Q_{t}=(0, t) \times \Omega . h=h(t, x)$ is the height that is measured in a co-moving frame of reference and describes the local position of the moving surface. $\nabla_{x} h$ is the gradient of $h$, and $\Delta_{x}=\partial^{2} / \partial x_{1}^{2}+\partial^{2} / \partial x_{2}^{2}$ is the Laplacian. For simplicity of notations, we shall use the following notations

$$
\xi=(p, q)=\nabla_{x} h .
$$

Then the equation turns out to be

$$
\frac{\partial h}{\partial t}+\nu \Delta_{x}^{2} h+\operatorname{div}_{x}\left(J\left(\nabla_{x} h\right)\right)=0 .
$$

which is satisfied in $Q_{T}$, where $T$ is a given positive number. And the boundary and initial conditions are

$$
\begin{aligned}
\frac{\partial}{\partial n} h=0,\left(\nu \nabla_{x} \Delta_{x} h+J\left(\nabla_{x} h\right)\right) \cdot n=0, & \text { on }[0, T] \times \partial \Omega, \\
h(0, x)=h_{0}(x), & x \in \bar{\Omega} .
\end{aligned}
$$


Here $n=\left(n_{1}, n_{2}\right)$ is the unit outward normal vector to the boundary $\partial \Omega$. We have introduced the surface diffusion current $J=J\left(\nabla_{x} h\right)=J(p, q)$

$$
\begin{aligned}
J & =\left(j_{1}, j_{2}\right), \\
j_{1} & =\alpha\left((p+q) f\left((p+q)^{2}\right)+(p-q) f\left((p-q)^{2}\right)\right), \\
j_{2} & =\alpha\left((p+q) f\left((p+q)^{2}\right)-(p-q) f\left((p-q)^{2}\right)\right),
\end{aligned}
$$

where $f$ is defined by

$$
f(y)=\frac{1-y}{(1-y)^{2}+\beta y},
$$

and $\alpha$ is a constant of surface diffusion, $\beta=\left(\ell_{d}\right)^{2}$ where $\ell_{d}$ is the diffusion length.

This completes the formulation of an initial-boundary value problem. It is worth a remark on the nonlinearity $f$ since there are several varieties of $f$ which lead to different models relating to ours.

Remark 1. Define

$$
f(y)=\frac{1}{1+\beta y} .
$$

Then the corresponding model is proposed by Johnson et al. [9]. However this model does have a slope selection mechanism, and is correct only for early time as long as the slopes are much smaller than 1 . It is too restrictive to describe the unstable three-dimensional growth of real materials in the later time regime. Therefore, (1.7) is introduced by Siegert et al in [23] to interpolate the two regimes. The form of the surface current $J$ is the minimal model in the sense that the nonlinearity must be chosen such that it describes the instability and leads to slope selection. The flux still has the correct physical behavior: $|J| \sim \sqrt{p^{2}+q^{2}}$ for $p^{2}+q^{2} \ll \frac{1}{\ell_{d}}$ and $|J| \sim 1 / \sqrt{p^{2}+q^{2}}$ for $\frac{1}{\ell_{d}} \ll p^{2}+q^{2} \ll 1$. This type of fluxes gives rise a completely different behavior than the one defined by (1.8), despite many similarities, as shown in [23]. The exact form of $f$ does not play a role since the slope selection mechanism and the growth exponents do not depend on such details.

In $[24,20]$, the current of the form

$$
J=\xi\left(1-|\xi|^{2}\right)
$$

is used, however, it has stable zeros for all slopes with $|\xi|=1$ regardless of the direction of $\xi$. Thus such an azimuthal symmetry is unrealistic for crystalline films. Therefore here $f_{i}(i=1,2)$ are functions chosen such that $f_{1}\left(p^{2}, q^{2}\right)=f_{2}\left(q^{2}, p^{2}\right)$. The simplest form that describes growth on such substrates is a current with components

$$
\begin{aligned}
& j_{1}=p\left(1-p^{2}-b q^{2}\right), \\
& j_{2}=q\left(1-q^{2}-b p^{2}\right),
\end{aligned}
$$

which leads to a buildup of pyramids with selected slopes $\left(p_{0}, q_{0}\right)=( \pm 1, \pm 1) / \sqrt{1+b}$ for $-1<b<1$. This diffusion current is suitable for substrates with a quadratic symmetry. 
Finally we point out that after a coordinate transformation $X=A x$, where

$$
A=\left(\begin{array}{cc}
1 & 1 \\
1 & -1
\end{array}\right)
$$

we find $J$ in (1.7) can be reduced, without loss of generality, to a simpler form

$$
\begin{aligned}
& j_{1}=\alpha p f\left(p^{2}\right), \\
& j_{2}=\alpha q f\left(q^{2}\right) .
\end{aligned}
$$

It is interesting to compare the important difference between the Cahn-Hilliard equation modeling phase-ordering and the model considered here. Mathematically, equation (1.1), with nonlinearity (1.8), (1.9) or (1.13), differs from the Cahn-Hilliard equation due to the flux term: $J$ in this paper depends on the gradient of the unknown, while the Cahn-Hilliard equation on the unknown only. Numerical experiments also show the important differences between this model and the Cahn-Hilliard one. Many papers, e.g. $[23,22,21]$ have been carried out the study of the differences between the problem studied here and phase-ordering dynamics described by the Cahn-Hilliard equation [4]. These differences become apparent when the domain configurations are plotted as in Figure 1. A domain in this context is an area of constant slope corresponding to one of the four values. The analogous case in phase-ordering dynamics is described by a fourstate clock model, see, e.g. [21, 8]. However, in that case we shall find that domain walls do not have any particular orientation, whereas here domain walls are intersections of planes of constant slopes and therefore form straight lines. Furthermore, there are two types of domain walls: Domain walls at which only one component of the slope changes are aligned along the $x$ and $y$ axes. These are the edges of the pyramids; Domain walls at which both components of the slope change run at $45^{\circ}$ with respect to the principal axes. These latter domain walls form the so-called roof tops.
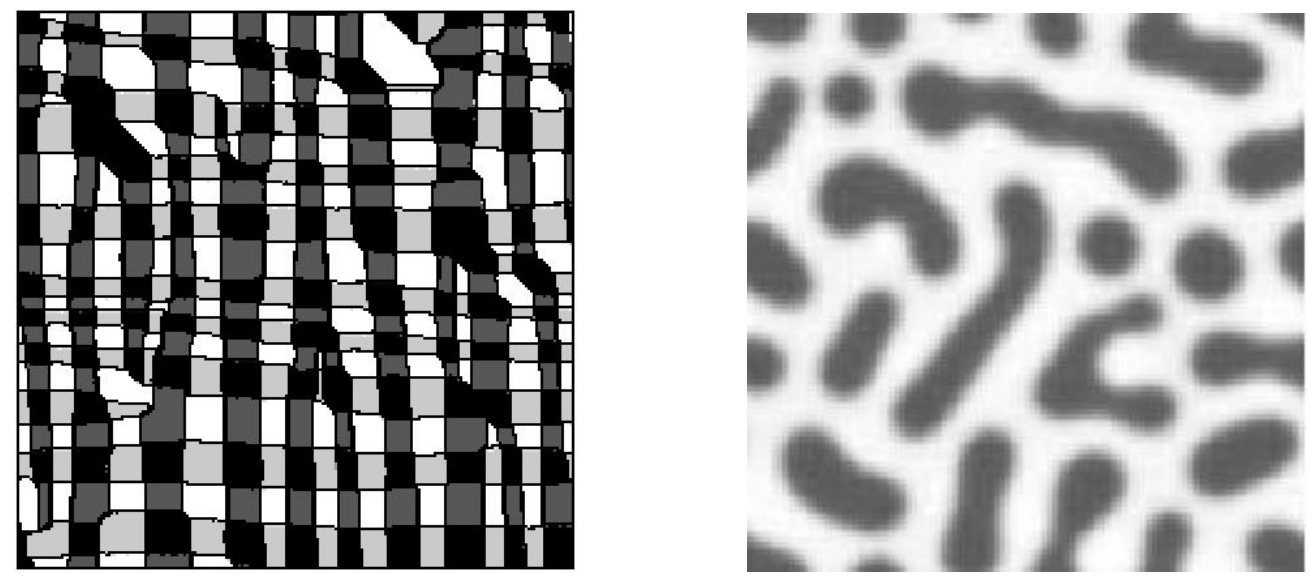

Figure 1. Left: the configuration for the crystal growth model, from [22]; Right: the domain walls of phase-ordering governed by the Cahn-Hilliard equation, from e.g. [8].

Now we derive the model briefly. Define the free energy by

$$
E[h]=\int_{\Omega}\left(\frac{\nu}{2}\left(\Delta_{x} h\right)^{2}-\frac{\alpha}{2}\left(F\left(\left(h_{x_{1}}+h_{x_{2}}\right)^{2}\right)+F\left(\left(h_{x_{1}}-h_{x_{2}}\right)^{2}\right)\right)\right) d x .
$$


Here $F=\int{ }^{x} f(y) d y$ is the primitive of $f$ such that $F(x)$ grows at $-\log (|x|)$ as $|x|$ goes to $\infty$. Suppose now that $h=h(t, x)$ is a solution to (1.1) - (1.3). Formal computations yield

$$
\begin{aligned}
& \frac{d}{d t} E[h]=\int_{\Omega}\left(-\alpha f\left(\left(h_{x_{1}}+h_{x_{2}}\right)^{2}\right)\left(h_{x_{1}}+h_{x_{2}}\right)\left(h_{x_{1}}+h_{x_{2}}\right)_{t}\right) d x \\
& +\int_{\Omega}\left(-\alpha f\left(\left(h_{x_{1}}-h_{x_{2}}\right)^{2}\right)\left(h_{x_{1}}-h_{x_{2}}\right)\left(h_{x_{1}}-h_{x_{2}}\right)_{t}+\nu \Delta_{x} h \Delta_{x} h_{t}\right) d x .
\end{aligned}
$$

Combining the terms containing $\left(h_{x_{1}}\right)_{t}\left(\right.$ or $\left.\left(h_{x_{2}}\right)_{t}\right)$ together we can rewrite the right hand side of the above equality as

$$
\begin{aligned}
& -\int_{\Omega} \alpha\left(f\left(\left(h_{x_{1}}+h_{x_{2}}\right)^{2}\right)\left(h_{x_{1}}+h_{x_{2}}\right)+f\left(\left(h_{x_{1}}-h_{x_{2}}\right)^{2}\right)\left(h_{x_{1}}-h_{x_{2}}\right)\right)\left(h_{x_{1}}\right)_{t} d x \\
& -\int_{\Omega} \alpha\left(f\left(\left(h_{x_{1}}+h_{x_{2}}\right)^{2}\right)\left(h_{x_{1}}+h_{x_{2}}\right)-f\left(\left(h_{x_{1}}-h_{x_{2}}\right)^{2}\right)\left(h_{x_{1}}-h_{x_{2}}\right)\right)\left(h_{x_{2}}\right)_{t} d x \\
& +\int_{\Omega} \nu \Delta_{x} h \Delta_{x} h_{t} d x \\
= & \int_{\Omega}\left(-J \cdot \nabla_{x} h_{t}+\nu \Delta_{x} h \Delta_{x} h_{t}\right) d x
\end{aligned}
$$

Using integration by parts and equation (1.1), we infer from (1.14) that

$$
\begin{aligned}
\frac{d}{d t} E[h] & =\int_{\Omega}\left(-J-\nu \nabla_{x} \Delta_{x} h\right) \cdot \nabla_{x} h_{t} d x \\
& =\int_{\Omega} \operatorname{div}_{x}\left(J+\nu \nabla_{x} \Delta_{x} h\right) h_{t} d x \\
& =-\int_{\Omega}\left(\operatorname{div}_{x}\left(J+\nu \nabla_{x} \Delta_{x} h\right)\right)^{2} d x \\
& \leq 0 .
\end{aligned}
$$

This implies the second law of thermodynamics is valid. The equation can be written in a gradient form with the total surface current defined by $J_{1}:=J_{e q}+J\left(\nabla_{x} h\right)$ where $J_{e q}:=\nu \nabla_{x} \Delta_{x} h$ is called the equilibrium surface current, $\nu$ is proportional to the surface stiffness.

Statement of the main result. Before the statement of our main results, we define weak solutions to problem consisting of (1.1) - (1.3). We use the notations: $(f, g)_{Q_{T}}$ and $(f, g)_{\Omega}$ are, respectively, the inner product of $f$ and $g$ over $Q_{T}$ and $\Omega$. $\langle f, g\rangle_{Q_{T}}$ denotes the dual product of $f, g$ with $f \in L^{2}\left(0, T ; X^{\prime}\right), g \in L^{2}(0, T ; X)$ and $X$ is a Banach space and $X^{\prime}$ is its dual. $H^{m}(\Omega)$ are the standard Hilbert spaces of order $m$. Define $H_{N}^{2}(\Omega):=\left\{f \in H^{2}(\Omega) \mid \frac{\partial f}{\partial n}=0\right.$ on $\left.\partial \Omega\right\}$ and its dual space is denoted by $H_{N}^{-2}(\Omega)$.

Definition 1.1 Let $h_{0} \in L^{2}(\Omega)$. A function $h=h(t, x)$ with

$$
h \in L^{\infty}\left(0, T ; L^{2}(\Omega)\right) \cap L^{2}\left(0, T ; H^{2}(\Omega)\right), h_{t} \in L^{2}\left(0, T ; H_{N}^{-2}(\Omega)\right)
$$

is a weak solution of problem (1.1) - (1.3), if

$$
\left\langle h_{t}, \varphi\right\rangle_{Q_{T}}+\nu\left(\Delta_{x} h, \Delta_{x} \varphi\right)_{Q_{T}}+\left(J, \nabla_{x} \varphi\right)_{Q_{T}}=0
$$

holds for all $\varphi \in L^{2}\left(0, T ; H_{N}^{2}(\Omega)\right)$, and $\lim _{t \downarrow 0^{+}}(h(t), \psi)_{\Omega}=\left(h_{0}, \psi\right)_{\Omega}$ for all $\psi \in L^{2}(\Omega)$. 
Now we are in a position to state the main results of this article.

Theorem 1.2 (Existence) Suppose that the boundary of $\Omega$ is smooth, and $h_{0} \in$ $L^{2}(\Omega)$. Then there exists a unique weak solution $h$ of problem $(1.1)-(1.3)$ in the sense of Definition 1.1, and the total mass $\int_{\Omega} h(t, x) d x$ is conserved, i.e. $\int_{\Omega} h(t, x) d x \equiv$ $\int_{\Omega} h_{0}(x) d x$

Moreover, if $h_{0} \in H^{1}(\Omega)$, the weak solution of problem (1.1) - (1.3) has, which in addition to (1.16), the following regularities

$$
h \in L^{\infty}\left(0, T ; H^{1}(\Omega)\right) \cap L^{2}\left(0, T ; H^{3}(\Omega)\right), h_{t} \in L^{2}\left(0, T ; H^{-1}(\Omega)\right) .
$$

Theorem 1.3 (Regularity) Suppose that $h_{0} \in H^{2 m}(\Omega)$ with $m \in \mathbb{N}$. Then the weak solution $h$ of problem (1.1) - (1.3) satisfies

$$
h \in L^{\infty}\left(0, T ; H^{2 m}(\Omega)\right) \cap L^{2}\left(0, T ; H^{2 m+2}(\Omega)\right), h_{t} \in L^{2}\left(0, T ; H^{2 m-2}(\Omega)\right),
$$

and

$$
\begin{array}{ll}
D_{t}^{l} h \in L^{\infty}\left(0, T ; L^{2}(\Omega)\right) \cap L^{2}\left(0, T ; H^{2}(\Omega)\right), & \text { if } m=2 l, l \in \mathbb{N} ; \\
D_{t}^{l-1} h \in L^{\infty}\left(0, T ; H^{2}(\Omega)\right), D_{t}^{l} h \in L^{2}\left(0, T ; L^{2}(\Omega)\right) & \text { if } m=2 l-1, l \in \mathbb{N} .
\end{array}
$$

Consequently, if $h_{0} \in C^{\infty}(\bar{\Omega})$, then the solution $h$ is smooth on $\bar{Q}_{T}$.

Now let us recall some references related closely to our problem. In [11] an initial boundary value problem of this epitaxial model with cubic nonlinearities is studied in which the initial data is chosen in $H^{2}(\Omega)$, and the $H^{2}(\Omega)$-norm of the solution follows directly from the Clausius-Duhem inequality, the second laws of thermodynamics, but this technique does not work for our case since we assume the initial data is only in $H^{1}(\Omega)$. Li and Liu study the initial boundary value problem for the MBE model with or without slope selection in [17] and the boundary conditions are chosen periodic. In both articles, they construct approximate solutions by using the Galerkin method, while we use a linearized problem, together with the convolution technique, to obtain a sequence of smooth approximate solutions, then establish a priori estimates for this sequence. Kohn and Otto [12] investigate the coarsening Rate for the Cahn-Hilliard equation, and Kohn and Yan [13] studies the coarsening rate for an epitaxial growth model with a cubic nonlinearity. Watson and Norris [27] study the coarsening dynamics of multiscale solutions to a dissipative singularly perturbed partial differential equation with a trigonally symmetric potential which models the evolution of a thermodynamically unstable crystalline surface.

We give now a remark on the choice of the initial data in this article.

Remark 2. The assumption that initial data $h_{0}$ is in $H^{1}$ is more natural than the one that $h_{0}$ is in $H^{2}$. The reason is that $h$ is piecewise affine in the case that the surfaces are high-symmetric, such as pyramidlike ones. One evidence can be also seen from a typical Scanning Tunneling Microscope (STM) picture, see Figure 2, which shows clearly that the surface are not smooth. Correspondingly, a good mathematical model should consider this feature.

If the initial data is in $H^{1}$, the existence of weak solutions to problem (1.1) - (1.3) in which the nonlinearity is cubic, like (1.9), also (1.10) - (1.11), is still open, and may 
be interesting. To solve such a problem, I surmise we need to invent an inequality of the Brezis-Gallouet type [3].

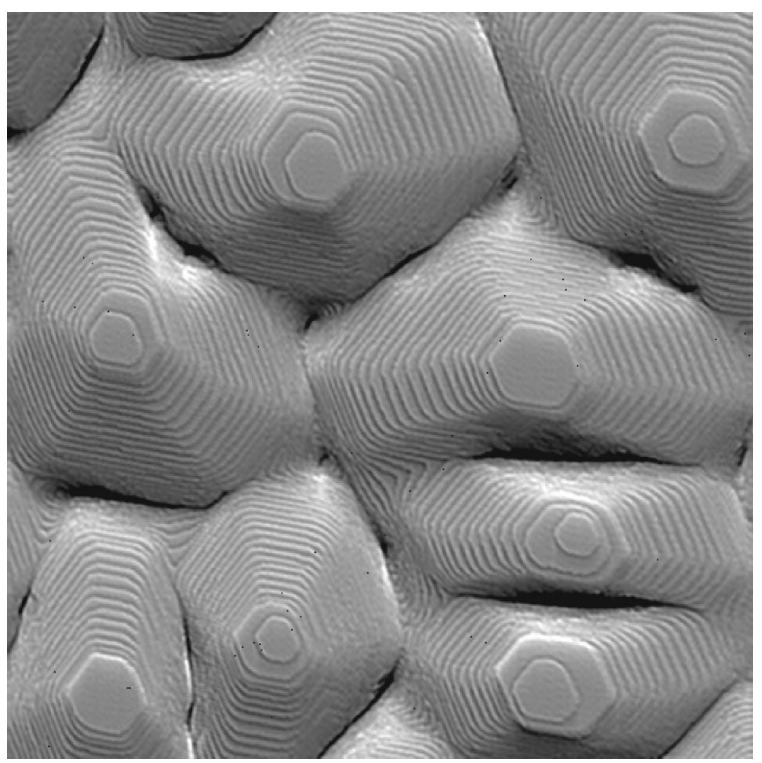

Figure 2. Typical STM image of mounds appearing on the surface. From Krug, Politi and Michley [15].

The organization of the remaining parts of this article is as follows. The existence and uniqueness of weak solutions is studied in Section 2 by constructing smooth approximate solutions and using a priori estimates. Section 3 consists of two parts, each of which is concerned, respectively, with the regularities of weak solutions to models with and without slope selection.

\section{Existence of weak solutions}

\subsection{Existence for the approximate problem}

To prove the existence Theorem 1.2, we construct smooth approximate solutions in a similar way as done in [1], provided the initial data is smooth. Then we establish uniform a priori estimates of these solutions by which we conclude compactness. Before formulating an approximate problem, we introduce the modifier $\chi=\chi(t, x)$ such that $\chi \in C_{0}^{\infty}\left(Q_{T}\right)$ satisfies $\int_{\mathbb{R}^{3}} \chi(t, x) d t d x=1$. For $\varepsilon>0$, we set

$$
\chi_{\varepsilon}(t, x):=\frac{1}{\varepsilon} \chi\left(\frac{t}{\varepsilon}, \frac{x}{\varepsilon}\right),
$$

and for any function $f \in L^{\infty}\left(Q_{T}\right)$ we define

$$
\widetilde{f}(t, x)=\left(\chi_{\varepsilon} * f\right)(t, x)=\int_{\mathbb{R}^{3}} \chi_{\varepsilon}(t-s, x-y) f(s, y) d s d y .
$$


We choose a smooth sequence $h_{0}^{\varepsilon}$ such that

$$
\left\|h_{0}^{\varepsilon}-h_{0}\right\|_{X} \rightarrow 0
$$

as $\varepsilon \rightarrow 0$. Here $X=L^{2}(\Omega)$ or $X=H^{1}(\Omega)$.

Then the smoothed problem turns out to be

$$
\left.\frac{\partial h}{\partial t}+\nu \Delta_{x}^{2} h+\operatorname{div}_{x}\left(J \widetilde{\nabla_{x} \hat{h}}\right)\right)=0,
$$

with the following boundary and initial conditions

$$
\begin{aligned}
& \frac{\partial}{\partial n} h=0, \quad\left(\nu \nabla_{x} \Delta_{x} h+J\left(\widetilde{\nabla_{x} \hat{h}}\right)\right) \cdot n=0, \quad \text { on }[0, T] \times \partial \Omega, \\
& h(0, x)=h_{0}^{\varepsilon}(x), \quad x \in \bar{\Omega} .
\end{aligned}
$$

Note that equation (2.2) is a linear fourth order parabolic equation with a smooth known term. By the existence theorem for higher order parabolic equations in the book by Ladysenskaya et al. [16] or the book by Eidelman [6], we have

Theorem 2.1 (Existence of smooth approximate solutions) Suppose that the assumptions of Theorem 1.2 are satisfied, and $\varepsilon$ is a given positive constant. Let $\hat{h} \in$ $L^{2}\left(0, T ; H^{2}(\Omega)\right)$.

Then for any $T>0$, there exists a unique smooth solution $h$ of problem $(2.2)-(2.4)$, which satisfies that the total mass $\int_{\Omega} h(t, x) d x$ is conserved.

The solution $h$ constructed in Theorem 2.1 depends on the small parameter $\varepsilon$. In order to prove the existence of weak solutions to the original problem (1.1) - (2.4), we need to establish some a priori estimates which are independent of $\hat{h}$ and $\varepsilon$ and thus guarantee the passage to limit of $h^{\varepsilon}$ as $\varepsilon \rightarrow 0$.

\section{$2.2 \quad$ A priori estimates}

Assume that there exists a classical solution $h^{\varepsilon}$ to problem $(2.2)-(2.4)$ with smooth initial data $h_{0}^{\varepsilon}$ and $\hat{h} \in L^{2}\left(0, T ; H^{2}(\Omega)\right)$ satisfying $\|\hat{h}\|_{L^{2}\left(0, T ; H^{2}(\Omega)\right)} \leq \bar{C}$. In what follows, $C$ denotes a constant which is independent of $\varepsilon$ and $\hat{h}$. The $L^{2}(\Omega)$-norm of $f$ is denoted by $\|f\|$. We shall derive a priori estimates for this solution. To begin with, we first state the following lemma on the nonlinearity $f$.

Lemma 2.1 There hold, for all $y \geq 0$, that

$$
\begin{aligned}
|y f(y)| & \leq C, \\
\left|y^{m+1} f^{(m)}(y)\right| & \leq C, m \in \mathbb{N} .
\end{aligned}
$$

Here $f^{(m)}$ is the $m$-th order derivative of $f$.

Proof. One needs to investigate the behavior of the function

$$
-y f(y)=\frac{y^{2}-y}{(1-y)^{2}+\beta y}
$$


for all $y \geq 0$. It is easy to see that $-y f(y) \rightarrow 1$ as $y \rightarrow \infty$, hence $|-y f(y)| \leq C$ for $y \geq M$, where $M$ is a suitably large constant. We shall prove that $-y f(y)$ is also bounded on the interval $[0, M]$. To this end, we need to prove that the denominator $g(y)=(1-y)^{2}+\beta y$ is greater than a positive constant. Note that $g(y)$ is nonnegative for $y \geq 0$. Thus 0 is the only possible minimum of $g(y)$. However $g(y)=0$ implies that $1-y=0$ and $y=0$ which cannot be satisfied simultaneously. Therefore, the minimum of $g$ must be positive, and since $M$ is finite, we infer from the continuity of $g(y)$ that

$$
\min _{0 \leq y \leq M} g(y)=C_{1}>0 .
$$

We then obtain, for all $y \in[0, M]$, that

$$
|y f(y)| \leq \frac{M^{2}+M}{C} \leq C .
$$

Next we consider the behavior of derivatives of $f$. Let $m \leq 1$ be an integer. Rewrite

$$
f^{(m)}(y)=((1-y) g(Y(y)))^{(m)}
$$

where $g(Y)=Y^{-1}$ and $Y=(1-y)^{2}+\beta y$. Invoking the product rule

$$
f_{0}^{(m)}=\left(f_{1} \cdot f_{2}\right)^{(m)}=\sum_{k=0}^{m} C_{m}^{k} f_{1}^{(k)} f_{2}^{(m-k)},
$$

where $C_{m}^{k}$ denotes the number of $k$-combinations of an $m$-element set, we have

$$
\begin{aligned}
f^{(m)}(y) & =C_{m}^{0}(1-y)(g(Y(y)))^{(m)}+C_{m}^{1}(1-y)^{\prime}(g(Y(y)))^{(m-1)} \\
& =(1-y)(g(Y(y)))^{(m)}-m(g(Y(y)))^{(m-1)} .
\end{aligned}
$$

Making use of the Faà di Bruno formula, i.e.

$$
\frac{d^{m}}{d x^{m}} g(Y(y))=\sum_{1 l_{1}+2 l_{2}+\cdots+m l_{m}=m} \frac{m !}{l_{1} ! l_{2} ! \cdots l_{m} !} g^{\left(l_{1}+l_{2}+\cdots+l_{m}\right)}(Y(y)) \prod_{j=1}^{m}\left(\frac{Y^{(j)}(y)}{j !}\right)^{l_{j}}
$$

and recalling $(g(Y))^{(m)}=\left(Y^{-1}\right)^{(m)}=(-1)^{m} m ! Y^{-m-1}, Y^{\prime}(y)=-2(1-y)+\beta$, $Y^{\prime \prime}(y)=2$ and $Y^{(j)}(y)=0$ for any $j>2$, we can reduce (2.9) to

$$
\frac{d^{m}}{d x^{m}} g(Y(y))=\sum_{1 l_{1}+2 l_{2}=m}(-1)^{l_{1}+l_{2}} \frac{m !\left(l_{1}+l_{2}\right) !}{l_{1} ! l_{2} !} Y^{-\left(l_{1}+l_{2}+1\right)}(y) \prod_{j=1}^{2}\left(\frac{Y^{(j)}(y)}{j !}\right)^{l_{j}} .
$$

For $y \in[0, M], f^{(m)}(y)$ is smooth by (2.7). One thus needs only to investigate the behavior for large $y \in[M, \infty)$, and it is enough to calculate the highest exponent. From (2.10) it follows that the highest exponent is less than or equal to

$$
-2\left(l_{1}+l_{2}+1\right)+1 l_{1}+0 l_{2}=-l_{1}-2 l_{2}-2=-m-2 .
$$

Therefore, invoking (2.8), we assert that there exists a constant $\gamma$ such that

$$
f^{(m)}(y) \sim \gamma y^{-m-1} \text { as } y \rightarrow \infty .
$$

Hence, this implies (2.6). Thus the proof of this lemma is complete.

From now on we are going to derive a priori estimates. The first is 
Lemma 2.2 There hold for all $t \in[0, T]$

$$
\begin{aligned}
\int_{\Omega} h(t, x) d x & =\int_{\Omega} h_{0}^{\varepsilon}(x) d x, \\
\|h(t)\|^{2}+\int_{0}^{t}\left\|\Delta_{x} h(\tau)\right\|^{2} d \tau & \leq C .
\end{aligned}
$$

Proof. Integrating (2.2) with respect to $x$ yields

$$
\frac{d}{d t} \int_{\Omega} h(t, x) d x=0
$$

which implies (2.12).

Multiplying (2.2) by $h$ and integrating the resulting equation with respect to $x$, we obtain

$$
\frac{1}{2} \frac{d}{d t}\|h\|^{2}+\nu\left\|\Delta_{x} h\right\|^{2}+\int_{\Omega} J\left(\widetilde{\nabla_{x} \hat{h}}\right) \cdot \nabla_{x} h d x=0 .
$$

Applying Lemma 2.1 we obtain

$$
\int_{\Omega} J\left(\widetilde{\nabla_{x} \hat{h}}\right) \cdot \nabla_{x} h d x \leq C\left\|\nabla_{x} h\right\| .
$$

Using the Poincaré inequality of the form $\left\|\nabla_{x} h\right\| \leq C\left\|D_{x}^{2} h\right\|+C\left(\int_{\Omega} h d x\right)$, applying the elliptic estimates $\left\|D_{x}^{2} h\right\| \leq C\left\|\Delta_{x} h\right\|$ and from (2.12) it then follows that

$$
\left\|\nabla_{x} h\right\| \leq C\left\|\Delta_{x} h\right\|+C .
$$

Therefore, (2.15) becomes

$$
\frac{1}{2} \frac{d}{d t}\|h\|^{2}+\nu\left\|\Delta_{x} h\right\|^{2} \leq \frac{\nu}{2}\left\|\Delta_{x} h\right\|^{2}+C .
$$

From this, estimate (2.13) follows. And the proof of this lemma is complete.

Lemma 2.3 There holds

$$
\|h(t)\|_{H^{1}(\Omega)}^{2}+\int_{0}^{t}\left\|\nabla_{x} \Delta_{x} h(\tau)\right\|^{2} d \tau \leq C .
$$

Proof. Integrating, with respect to $x$ over $\Omega$, equation (2.2) multiplied by $-\Delta_{x} h$ and using integration by parts yield

$$
\left.\frac{1}{2} \frac{d}{d t}\left\|\nabla_{x} h\right\|^{2}+\nu\left\|\nabla_{x} \Delta_{x} h\right\|^{2}-\int_{\Omega} J \widetilde{\left(\nabla_{x} \hat{h}\right.}\right) \cdot \nabla_{x} \Delta_{x} h d x=0
$$

Making use of Lemma 2.1 again, we can easily prove that

$$
\left|\int_{\Omega} J\left(\widetilde{\nabla_{x} \hat{h}}\right) \cdot \nabla_{x} \Delta_{x} h d x\right| \leq C+\frac{\nu}{2}\left\|\nabla_{x} \Delta_{x} h\right\|^{2} .
$$

Thus integrating (2.19) with respect to $t$ one gets

$$
\frac{1}{2}\left\|\nabla_{x} h\right\|^{2}+\nu \int_{0}^{t}\left\|\nabla_{x} \Delta_{x} h\right\|^{2} d \tau \leq C+\frac{\nu}{2} \int_{0}^{t}\left\|\nabla_{x} \Delta_{x} h\right\|^{2} d \tau .
$$

From which estimate (2.18) follows. The proof of this lemma is complete. 


\subsection{Existence of solutions to the phase field model}

In this section we shall make use of the a priori estimates established in the previous subsection to study the convergence of the solutions $h^{m}$ of the approximate problem for $m \rightarrow \infty$, thereby proving Theorem 1.2. In our investigation we need the following well known results, see, for instance, Lions [18], Evans [7]:

We shall make use of the following lemma which is of Aubin-Lions type.

Lemma 2.4 Let $B_{0}, B, B_{1}$ be Banach spaces which satisfy that $B_{0}, B_{1}$ are reflexive and that

$$
B_{0} \subset \subset B \subset B_{1} .
$$

Here, by $\subset \subset$ we denote the compact imbedding. Define

$$
W=\left\{f \mid f \in L^{\infty}\left(0, T ; B_{0}\right), \quad \frac{d f}{d t} \in L^{r}\left(0, T ; B_{1}\right)\right\}
$$

with $T$ being a given positive number and $1<r<\infty$.

Then the embedding of $W$ in $C([0, T] ; B)$ is compact.

To deal with the nonlinear terms, we also need

Lemma 2.5 Let $\Gamma$ be an open set in $\mathbb{R}^{m}$. Suppose functions $f_{n}, f$ are in $L^{q}(\Gamma)$ for any given $1<q<\infty$, which satisfy

$$
\left\|f_{n}\right\|_{L^{q}(\Gamma)} \leq C, f_{n} \rightarrow f \text { almost everywhere in } \Gamma .
$$

Then $f_{n}$ converges to $f$ weakly in $L^{q}(\Gamma)$.

We now turn to prove the existence of weak solutions for the initial $h_{0}$ that is assumed in $L^{2}(\Omega)$. The existence for the initial data in $H^{1}(\Omega)$ is easy by recalling Lemma 2.3. Using problem $(2.2)-(2.4)$, we can construct smooth approximate solutions as follows: Let $h^{1}$ be a given function. By solving problem $(2.2)-(2.4)$ with $\hat{h}=h^{1}$, one gets $h^{2}$. Suppose we have obtained $h^{m}$ for some $m \in \mathbb{N}$, then set $\varepsilon=\frac{1}{m}$ and $\hat{h}=h^{m}$. Thus, we can define $h^{m+1}$ successively. Therefore we has a sequence of approximate solutions $h^{m}$. Since equation (1.1) is nonlinear, we need some results about strong and pointwise convergence.

Define $f=h^{j}$ and $r=2$. Set

$$
B_{0}=H^{2}(\Omega), B=H^{1}(\Omega), B_{1}=H^{-2}(\Omega),
$$

it follows from Lemma 2.2 that

$$
\|h\|_{L^{2}\left(0, T ; B_{0}\right)} \leq C,\left\|h_{t}\right\|_{L^{2}\left(0, T ; B_{1}\right)} \leq C .
$$

Applying Lemma 2.4, we then conclude that $\left\{h^{m}\right\}$ is compact in $C([0, T] ; B)$, namely $C\left([0, T] ; H^{1}(\Omega)\right)$, so $\left\{h_{x_{i}}^{m}\right\}$, for $i=1,2$, is compact in $C\left([0, T] ; L^{2}(\Omega)\right)$. Therefore there exists a subsequence, still denote it by $\left\{h_{x_{i}}^{m}\right\}$, such that

$$
\left\|h^{m}-h\right\|_{C\left([0, T] ; H^{1}(\Omega)\right)} \rightarrow 0, \quad\left\|h_{x_{i}}^{m}-h_{x_{i}}\right\|_{C\left([0, T] ; L^{2}(\Omega)\right)} \rightarrow 0
$$


as $m \rightarrow \infty$. Moreover, we can select furthermore a subsequence such that $h_{x_{i}}^{m}$ converges to $h_{x_{i}}$ almost everywhere. Setting $\kappa=\frac{1}{m}, S^{\kappa}=\nabla_{x} h^{m}$. By the properties of convolution and (2.22), we have

$$
\begin{aligned}
\left\|\chi_{\kappa} * S^{\kappa}-S\right\|_{L^{2}\left(Q_{T_{e}}\right)} & \leq\left\|\chi_{\kappa} *\left(S^{\kappa}-S\right)\right\|_{L^{2}\left(Q_{T_{e}}\right)}+\left\|\left(S-\chi_{\kappa} * S\right)\right\|_{L^{2}\left(Q_{T_{e}}\right)} \\
& \leq\left\|\left(S-\chi_{\kappa} * S\right)\right\|_{L^{2}\left(Q_{T_{e}}\right)}+\left\|S^{\kappa}-S\right\|_{L^{2}\left(Q_{T_{e}}\right)} \rightarrow 0,
\end{aligned}
$$

for $\kappa \rightarrow 0$, whence we can select a subsequence, still denote by $\chi_{\kappa} * S^{\kappa}$ converges to $S$ almost everywhere. Consequently, we assert that

$$
J\left(\widetilde{\nabla_{x} h^{m}}\right) \text { converges to } J\left(\nabla_{x} h\right)
$$

almost everywhere as $m \rightarrow \infty$. Remembering that $\left\|J\left(\widetilde{\nabla_{x} h^{m}}\right)\right\|_{L^{2}\left(Q_{T}\right)} \leq C$, using Lemma 2.5 we assert that

$$
J\left(\widetilde{\nabla_{x} h^{m}}\right) \rightarrow J\left(\nabla_{x} h\right)
$$

in $L^{2}\left(Q_{T}\right)$ as $m \rightarrow \infty$.

For the linear terms, by weak compactness, one can easily get

$$
\left\langle h_{t}^{m}, \varphi\right\rangle \rightarrow\left\langle h_{t}, \varphi\right\rangle,\left(\Delta_{x} h^{m}, \Delta_{x} \varphi\right) \rightarrow\left(\Delta_{x} h, \Delta_{x} \varphi\right)
$$

as $m \rightarrow \infty$, for all $\varphi \in L^{2}\left(0, T ; H_{N}^{2}(\Omega)\right)$.

Taking the inner product of $(2.2)$ and $\varphi$ we arrive at

$$
\begin{aligned}
0 & =\left\langle\frac{\partial h^{m}}{\partial t}, \varphi\right\rangle+\nu\left(\Delta_{x} h^{m}, \Delta_{x} \varphi\right)-\left(J\left(\widetilde{\nabla_{x} h^{m}}\right), \nabla_{x} \varphi\right) \\
& \rightarrow\left\langle\frac{\partial h}{\partial t}, \varphi\right\rangle+\nu\left(\Delta_{x} h, \Delta_{x} \varphi\right)-\left(J\left(\nabla_{x} h\right), \nabla_{x} \varphi\right) .
\end{aligned}
$$

Thus (1.17) is proved. From (2.12), (2.22) and the choice of the smooth initial data $h_{0}^{\varepsilon}$ (let $\varepsilon=\frac{1}{m}$ ) we have

$$
\int_{\Omega} h^{m}(t, x) d x=\int_{\Omega} h_{0}^{\frac{1}{m}}(x) d x \rightarrow \int_{\Omega} h_{0}(x) d x,
$$

and the left hand side converges to $\int_{\Omega} h(t, x) d x$, thus the mass is conserved for weak solution. And the existence of weak solutions is complete.

Next we are going to study the

Stability and Uniqueness. Let $h_{1}, h_{2}$ be two weak solutions corresponding to initial data $h_{0}^{1}$ and $h_{0}^{2}$, respectively. Define $u=h_{1}-h_{2}$. We write

$$
\nabla_{x} h_{i}=\left(p_{i}, q_{i}\right),(i=1,2), \nabla_{x} u=(p, q), J_{i}=J\left(\nabla_{x} h_{i}\right) .
$$

Then by the estimates in Lemma 2.1 we have $\left|J_{1}-J_{2}\right| \leq C\left|\nabla_{x} h_{1}-\nabla_{x} h_{2}\right|=C\left|\nabla_{x} u\right|$, hence

$$
\begin{aligned}
0 & =\frac{1}{2} \frac{d}{d t}\|u\|^{2}+\nu\left\|\Delta_{x} u\right\|^{2}+\int_{\Omega}\left(J_{1}-J_{2}\right) \cdot \nabla_{x} u d x \\
& \geq \frac{1}{2} \frac{d}{d t}\|u\|^{2}+\nu\left\|\Delta_{x} u\right\|^{2}-C\left\|\nabla_{x} u\right\|^{2} .
\end{aligned}
$$


Making use of the Nirenberg inequality of the following form

$$
\left\|\nabla_{x} u\right\| \leq C\left\|\Delta_{x} u\right\|^{\frac{1}{2}}\|u\|^{\frac{1}{2}}+C^{\prime}\|u\|,
$$

and the Young inequality, from (2.28) one obtains

$$
\begin{aligned}
\frac{1}{2} \frac{d}{d t}\|u\|^{2}+\nu\left\|\Delta_{x} u\right\|^{2} & \leq C\left(\left\|\Delta_{x} u\right\|\|u\|+\|u\|^{2}\right) \\
& \leq \frac{\nu}{2}\left\|\Delta_{x} u\right\|^{2}+C\|u\|^{2} .
\end{aligned}
$$

Now using the Gronwall inequality we get

$$
\|u(t)\|^{2} \leq\|u(0)\|^{2} e^{C t} .
$$

Here $u(0)=h_{0}^{1}-h_{0}^{2}$. Thus the solution depends continuously on the initial data.

Consequently, if $h_{0}^{1}=h_{0}^{2}$, that is $\|u(t)\|^{2}=0$ which implies $\|u(t)\|^{2}=0$, so the weak solution is unique. Therefore, the proof of Theorem 1.3 is complete.

\section{Regularity of weak solutions}

We shall investigate the regularity of weak solutions to both models with and without slope selection, while for the latter model we can only carry out such study in one space dimension.

\subsection{The model with slope selection}

Suppose now that $h_{0} \in H^{2 m}(\Omega)$ with $m \in \mathbb{N}$ and there exists a unique solution $h$ to problem $(1.1)-(1.3)$. In this section we shall investigate the regularities of this solution. We first consider the case that $m=1$.

Lemma 3.1 There hold for $h_{0} \in H^{2}(\Omega)$ that

$$
\begin{aligned}
\|h(t)\|_{H^{2}(\Omega)}^{2}+\left\|h_{t}\right\|_{L^{2}\left(0, T ; L^{2}(\Omega)\right.}^{2} & \leq C, \\
\|h\|_{L^{2}\left(0, T ; H^{4}(\Omega)\right)}^{2} & \leq C .
\end{aligned}
$$

Proof. Multiplying equation (1.1) by $h_{t}$ and integrating the resulting equation with respect to $x$ yield

$$
\begin{aligned}
0 & =\left\|h_{t}\right\|^{2}+\frac{\nu}{2} \frac{d}{d t}\left\|\Delta_{x} h\right\|^{2}+\int_{\Omega} J \cdot \nabla_{x} h_{t} d x \\
& =\left\|h_{t}\right\|^{2}+\frac{d}{d t} E[h](t) .
\end{aligned}
$$

Thus one has

$$
\begin{aligned}
\int_{0}^{T}\left\|h_{t}\right\|^{2} d t & \leq C, \\
E[h](t) & \leq E[h](t) .
\end{aligned}
$$


Recalling the definitions of $E[h]$ and $f$ we arrive at (3.1).

From equation (1.1), making use the estimates in Lemma 2.1 and (3.1) we obtain

$$
\begin{aligned}
\int_{0}^{T}\left\|\Delta_{x}^{2} h(\tau)\right\|^{2} d \tau & \leq C \int_{0}^{T}\left\|h_{t}(\tau)\right\|^{2} d \tau+C \int_{0}^{T}\left\|D^{2} h\right\|^{2} d \tau, \\
& \leq C .
\end{aligned}
$$

Thus (3.2) is proved. And the proof of this lemma is complete.

To get the a priori estimates for higher order derivatives, we differentiate the equation with respect to $t$ to get

$$
\frac{\partial h_{t}}{\partial t}+\nu \Delta_{x}^{2} h_{t}+\operatorname{div}_{x}\left(\left(J\left(\nabla_{x} h\right)\right)_{t}\right)=0
$$

Such computations are formal. However by using the technique of difference quotient one can justify easily. In a similar way for deriving the estimates for $h$, we arrive at

Lemma 3.2 Suppose that $h_{0} \in H^{4}(\Omega)$, i.e. $m=2$. There holds for any $t \in[0, T]$ that

$$
\left\|h_{t}\right\|^{2}+\|h\|_{H^{4}(\Omega)}^{2}+\left\|h_{t}\right\|_{L^{2}\left(0, T ; H^{2}(\Omega)\right.}^{2} \leq C .
$$

Proof. Multiplying equation (3.4) by $h_{t}$ and integrating the resulting equation with respect to $x$ yield

$$
\begin{aligned}
0 & =\frac{1}{2} \frac{d}{d t}\left\|h_{t}\right\|^{2}+\nu\left\|\Delta_{x} h_{t}\right\|^{2}+\int_{\Omega} J_{t} \cdot \nabla_{x} h_{t} d x \\
& \geq \frac{1}{2} \frac{d}{d t}\left\|h_{t}\right\|^{2}+\nu\left\|\Delta_{x} h_{t}\right\|^{2}-C\left\|\nabla_{x} h_{t}\right\|^{2} .
\end{aligned}
$$

With the help of the Nirenberg inequality (2.29) one obtains from (3.6) and the Young inequality that

$$
\begin{aligned}
\frac{1}{2} \frac{d}{d t}\left\|h_{t}\right\|^{2}+\nu\left\|\Delta_{x} h_{t}\right\|^{2} & \leq C\left\|\Delta_{x} h_{t}\right\|\left\|h_{t}\right\|+C\left\|h_{t}\right\|^{2} \\
& \leq \frac{\nu}{2}\left\|\Delta_{x} h_{t}\right\|^{2}+C\left\|h_{t}\right\|^{2} .
\end{aligned}
$$

From which, by the Gronwall inequality, it follows that

$$
\left\|h_{t}\right\|^{2}+\nu \int_{0}^{T}\left\|\Delta_{x} h_{t}\right\|^{2} d t \leq C .
$$

Furthermore, one can get from equation (1.1) and Lemma 2.1 that

$$
\begin{aligned}
\left\|\Delta_{x}^{2} h\right\|^{2} & \leq C\left\|h_{t}\right\|^{2}+C\left\|\Delta_{x}^{2} h\right\|^{2} \\
& \leq C
\end{aligned}
$$

which implies that $h \in L^{\infty}\left(0, T ; H^{4}(\Omega)\right)$. The proof of this lemma is thus complete.

For the higher order derivatives with both $x$ and $t$, we have 
Lemma 3.3 Let $h_{0} \in H^{2 m}(\Omega)$. There holds for any $t \in[0, T]$ that

$$
\|h(t)\|_{H^{2 m}(\Omega)}^{2}+\|h\|_{L^{2}\left(0, T ; H^{2 m+2}(\Omega)\right.}^{2}+\left\|h_{t}\right\|_{L^{2}\left(0, T ; H^{2 m-2}(\Omega)\right.}^{2} \leq C,
$$

and

$$
\begin{aligned}
\left\|D_{t}^{l} h\right\|_{L^{\infty}\left(0, T ; L^{2}(\Omega)\right)}^{2}+\left\|D_{t}^{l} h\right\|_{L^{2}\left(0, T ; H^{2}(\Omega)\right.}^{2} & \leq C, \text { if } m=2 l, \\
\left\|D_{t}^{l-1} h\right\|_{L^{\infty}\left(0, T ; H^{2}(\Omega)\right)}^{2}+\left\|D_{t}^{l} h\right\|_{L^{2}\left(0, T ; L^{2}(\Omega)\right.}^{2} & \leq C, \text { if } m=2 l-1 .
\end{aligned}
$$

Proof. We employ the mathematical induction. From Lemma 3.1, it is easy to see that (3.10) and (3.12) are true for $m=1$ which implies $l=1$ too. By Lemma 3.2, estimate (3.11) holds when $m=2$. Assume that (3.10) is true for any $k \leq m \in \mathbb{N}$ and (3.11) and (3.12) are true respectively for even and odd $m$. Next we shall prove they are true for $k \leq m+1$ when $h_{0} \in H^{2(m+1)}(\Omega)$.

For the case that $m+1$ is even (resp. odd), differentiating $\frac{m+1}{2}$ (resp. $\frac{m}{2}$ ) times equation (1.1) with respect to $t$, letting $v=D_{t}^{\frac{m+1}{2}} h$ (resp. $v=D_{t}^{\frac{m}{2}} h$ ), repeating the argument of Lemma 3.2 (resp. Lemma 3.1) for this function $v$, and using the estimates in Lemma 2.1, we then conclude (3.10) holds for $m+1$, moreover, (3.11) (resp. (3.12)) is true for $l=\frac{m+1}{2}$ (resp. $\left.l=\frac{m}{2}\right)$. Thus the proof of this lemma is complete.

\subsection{The one-dimensional cubic model without slope selection}

In this subsection we shall study the one-dimensional problem with a cubic current, i.e. (1.9), or $(1.10)-(1.11)$, which is studied in $[24,20,27]$. However the original two dimensional problem is still open. The problem is

$$
\begin{aligned}
h_{t}+\nu h_{x x x x}+\left(J\left(h_{x}\right)\right)_{x} & =0 \text { in } Q_{T}, \\
h_{x}=0, h_{x x x} & =0 \text { on }[0, T] \times \partial \Omega, \\
\left.h\right|_{t=0} & =h_{0} .
\end{aligned}
$$

Here $\Omega=(a, b) \subset \mathbb{R}, a, b \in \mathbb{R}$ and $J=\alpha h_{x}\left(1-h_{x}^{2}\right)$.

Assume that $h_{0} \in L^{2}(\Omega)$. Multiplying (3.13) by $h$ and integrating it with respect to $x$ give

$$
\begin{aligned}
0 & =\frac{1}{2} \frac{d}{d t}\|h\|^{2}+\nu\left\|h_{x x}\right\|^{2}-\int_{\Omega} J\left(h_{x}\right) h_{x} d x \\
& =\frac{1}{2} \frac{d}{d t}\|h\|^{2}+\nu\left\|h_{x x}\right\|^{2}+\int_{\Omega}\left|h_{x}\right|^{4} d x-\left\|h_{x}\right\|^{2},
\end{aligned}
$$

By the Young inequality $a^{2} \leq \frac{1}{2} a^{4}+\frac{1}{2}$, we infer from (3.16) that

$$
\frac{1}{2} \frac{d}{d t}\|h\|^{2}+\nu\left\|h_{x x}\right\|^{2}+\int_{\Omega}\left|h_{x}\right|^{4} d x \leq \frac{1}{2} \int_{\Omega}\left|h_{x}\right|^{4} d x+\frac{1}{2},
$$

which gives

Lemma 3.4 We have

$$
\|h\|^{2}+\int_{0}^{T}\left(\|h(t)\|_{H^{2}(\Omega)}^{2}+\int_{\Omega}\left|h_{x}\right|^{4} d x\right) d t \leq C .
$$


Based on this lemma we can define weak solutions and prove the existence and uniqueness in a similar way as in Section 2 for $h_{0} \in L^{2}(\Omega)$. Suppose that $h_{0} \in H^{1}(\Omega)$, is the weak solution regular, i.e. $h \in L^{2}\left(0, T ; H^{1}(\Omega)\right)$ ? To answer this question, we need more estimates. From equation (3.13), we obtain

$$
\begin{aligned}
0 & =\frac{1}{2} \frac{d}{d t}\left\|h_{x}\right\|^{2}+\nu\left\|h_{x x x}\right\|^{2}-\int_{\Omega} J\left(h_{x}\right)_{x} h_{x x} d x, \\
& =\frac{1}{2} \frac{d}{d t}\left\|h_{x}\right\|^{2}+\nu\left\|h_{x x x}\right\|^{2}+\int_{\Omega} 3\left|h_{x}\right|^{2}\left|h_{x x}\right|^{2} d x-\left\|h_{x x}\right\|^{2} .
\end{aligned}
$$

Therefore,

$$
\left\|h_{x}\right\|^{2}+\int_{0}^{T}\left(\nu\left\|h_{x x x}\right\|^{2}+\int_{\Omega} 3\left|h_{x}\right|^{2}\left|h_{x x}\right|^{2} d x\right) d t \leq C .
$$

So the weak solution is more regular if $h_{0} \in H^{1}(\Omega)$.

However for two-dimensional problem, we can not get (3.19) so that we obtain the good term $\int_{\Omega} 3\left|h_{x}\right|^{2}\left|h_{x x}\right|^{2} d x$ in (3.20).

Acknowledgement. The first author (JZ) of this work has been partly supported National Natural Science Foundation of China (Grant No. 10501040) and by Zhejiang Provinicial Natural Science Foundation of China (Grant No. Y6100611), and the second (PZ) by Grant MTM2008-03541 of the Ministerio de Educacíon y Ciencia of Spain, and by Project PI2010-04 of the Basque Government. The second author would like to express his sincere thanks to Dr. S. Watson at Univ. of Glasgow for bringing the paper [27] to the authors' attention.

\section{References}

[1] H.-D. Alber and P. Zhu, Solutions to a Model for interface motion by with interface deffusion, Proc. Roy. Soc. Edinburgh 138A, (2008) 923-955.

[2] A. Barabási and H. Stanley, Fractal concepts in surface growth, Cambridge Univ. Press, 1995.

[3] H. Brezis and T. Gallouet, Nonlinear Schrödinger evolution equations, Nonlinear Anan., Theo. Meth. \& Appl. 4 No. 4, (1980) 677-681.

[4] J. Cahn and J. Hilliard, Free Energy of a Nonuniform System. I. Interfacial Free Energy, J. Chem. Phys. Vol. 28, (1958) 258-267.

[5] M. Eden A probabilistic model for morphogenesis, in Symposium on information theory in biology 359-370. Edited by H. Yockey. Pergamon, New York, 1958.

[6] Eidelman, S. D., Parabolic systems, North-Holland, Amsterdam, 1969.

[7] L. Evans, Weak convergence methods for nonlinear partial differential equations, CBMS Regional conference Series in Math. 74, Amer. Math. Soc., Providence, 1990. 
[8] L. Foret, A simple mechanism of raft formation in two-component fluid membranes, Europhysics Letters, 71 No. 3, (2005) 508-514.

[9] M. Johnson, C. Orme, A. Hunt, D. Graff, J. Sudijono, L. Sander and B. Orr, Stable and unstable growth in molecular beam epitaxy, Phys. Review Letters, 72 No. 1, (1994) 116-119.

[10] M. Kardar, G. Parisi and Y. Zhang, Dynamical scaling of growing interfaces, Phys. Rev. Lett. 56 No 9, (1986) 889-892.

[11] B. King, O. Stein and M. Winkler, A fourth-order parabolic equation modeling epitaxial thin film growth, 286 (2003) 459-490.

[12] R. Kohn and F. Otto, Upper bounds on coarsening rates, Commun. Math. Phys. 229 (2002) 375-395.

[13] R. Kohn and X. Yan, Upper bounds on coarsening rates for an epitaxial growth model, Commun. Pure Appl. Math. LVI (2003) 1549-1564.

[14] J. Krug, Original of scal invariance in growth processes, Adv. Phys. 46 No. 2, (1997) 139-282.

[15] J. Krug, P. Politi and T. Michely, Island nucleation in the presence of step-edge barriers: Theory and applications Phys. Rev. B 61 No. 20, (2000) 14 037-14 046.

[16] O. Ladyzenskaya, V. Solonnikov and N. Uralceva, Linear and quasilinear equations of parabolic type, Translations of Mathematical Monographs 23, Providence: Amer. Math. Soc. 1968.

[17] B. Li and J. Liu, Thin film epitaxy with or without slope selection Euro. J. Appl. Math. 14 (2003) 713-743.

[18] J. Lions, Quelques methodes de resolution des problemes aux limites non lineaires, Dunod Gauthier-Villars, Paris 1969.

[19] M. Plischke, Z. Rácz and D. Liu, Time-reversal invariance and universality of twodimensional growth models Phys. Rev. B 35 (1987) 3485-3495.

[20] M. Rost and J. Krug, Coarsening of surface structures in unstable epitaxial growth, Phys. Rev. E 55 No. 4, (1997) 3952-3957.

[21] M. Siegert, Ordering dynamics of surfaces in molecular beam epitaxy, Physica A 239 (1997) 420-427.

[22] M. Siegert, Coarsening dynamics of crystalline thin films, Phys. Rev. Lett. 81 No. $25,(1998) 5481-5484$.

[23] M. Siegert and M. Plischke, Slope selection and coarsening in molecular beam epitaxy, Phys. Rev. Lett. 73 No. 11, (1994) 1517-1520. 
[24] J. Stroscio, D. Pierce, M. Stiles, A. Zangwill and L. Sander, Coarsening of unstable surface features during $\mathrm{Fe}(001)$ homoepitaxy, Phys. Rev. Lett. 75 No. 23, (1995) $4246-4249$.

[25] J. Villain, Continuum models of crystal growth from atomic beams with and without desorption J. Phys. I 1 (1991) 19-42.

[26] M. Vold, A numerical approach to the problem of sediment volume, J. Colloid Sci. 14 (1959) 168-174.

[27] S.Watson and S. Norris, Scaling Theory and Morphometrics for a Coarsening Multiscale Surface, via a Principle of Maximal Dissipation, Phys. Rev. Lett. 96 (2006) 176103-1 - 176103-4. 